\title{
Automated Reasoning and Presentation Support for Formalizing Mathematics in Mizar
}

\author{
Josef Urban ${ }^{1 \star \star}$ and Geoff Sutcliffe ${ }^{2}$ \\ 1 Radboud University, Nijmegen \\ 2 University of Miami
}

\begin{abstract}
This paper presents a combination of several automated reasoning and proof presentation tools with the Mizar system for formalization of mathematics. The combination forms an online service called MizAR, similar to the SystemOnTPTP service for first-order automated reasoning. The main differences to SystemOnTPTP are the use of the Mizar language that is oriented towards human mathematicians (rather than the pure first-order logic used in SystemOnTPTP), and setting the service in the context of the large Mizar Mathematical Library of previous theorems, definitions, and proofs (rather than the isolated problems that are solved in SystemOnTPTP). These differences poses new challenges and new opportunities for automated reasoning and for proof presentation tools. This paper describes the overall structure of MizAR, and presents the automated reasoning systems and proof presentation tools that are combined to make MizAR a useful mathematical service.
\end{abstract}

\section{Introduction and Motivation}

Formal mathematics, in its interactive and verification aspects, and in the automated reasoning aspect, is becoming increasingly well-known, used, and experimented with [10]. Projects like FlySpeck [9], formal proof of the Four Color Theorem [8], verification of tiny (but real) operating systems [12], and the increased use of verification for software and hardware [7], are stimulating the development of interactive verification tools and interactive theorem provers (ITPs). Linked to this is the development of strong automated theorem proving (ATP) systems, used either independently to solve hard problems in suitable domains $[14,17,3]$, or integrated with interactive tools $[15,11,4]$. ATP development has also stimulated interesting research in the context of automated reasoning in large theories $[16,34,21]$.

The goal of the work presented here is to make formal mathematics and automated reasoning easily accessible to practitioners in these areas, by putting most of the work into their browsers, and providing a very fast (real-time) server-based experience with a number of ATP, ITP, presentation, and AI tools that work

\footnotetext{
* The final publication of this paper is available at www.springerlink.com

** Supported by the NWO project "MathWiki a Web-based Collaborative Authoring Environment for Formal Proofs".
} 
well together. This is important for supporting existing users and attracting new users of Mizar, by providing them with an attractive environment for exploring the world of formal reasoning in mathematics. Fast server-based solutions make systems easy to use, to the extent of just "pushing a button" (clicking on a HTML link), rather than having to go through the pains of building an adequate local hardware and software installation, for benefits that might initially not be clear. Server-based solutions are becoming an important part of general computer use, and formal mathematics is no exception. It is not possible to name all the server-based services that already exist for informal mathematics, starting e.g., from the arXiv, Wikipedia, MathOverflow, PolyMath, Wolfram MathWorld, PlanetMath, ProofWiki, the SAGE system for working with CASes, etc.

This paper describes the Automated Reasoning for Mizar (MizAR) web service, which combines several automated reasoning and proof presentation tools with the Mizar system for formalization of mathematics, to form a useful mathematical service. MizAR runs in the context of the Mizar Mathematical Library (MML), and uses the Mizar language that is oriented towards human mathematicians. The main inspiration for MizAR is the SystemOnTPTP ATP service [22]. SystemOnTPTP allows users to easily experiment with many first-order ATP systems in a common framework, and provides additional services such as proof presentation with the IDV system [28], discovery of interesting lemmas with the AGInT system [18], and independent proof verification with the GDV verifier [23]. Pieces of the SystemOnTPTP infrastructure also served in the initial implementation of the MizAR web service. SystemOnTPTP's infrastructure is briefly described in Section 2. Section 3 describes the implemented MizAR service, and demonstrates its use. Section 4 considers a number of possible future extensions, and concludes.

\section{SystemOnTPTP}

The core of SystemOnTPTP is a utility that allows an ATP problem or solution to be easily and quickly submitted in various ways to a range of ATP systems and tools. SystemOnTPTP uses a suite of currently available systems and tools, whose properties (input format, reporting of result status, etc) are stored in a simple text database. The input can be selected from the TPTP (Thousands of Problems for Theorem Provers) problem library or the TSTP (Thousands of Solutions from Theorem Provers) solution library [24], or provided in TPTP format [25] by the user. The implementation relies on several subsidiary tools to preprocess the input, control the execution of the chosen ATP system(s), and postprocess the output. On the input side TPTP2X or TPTP4X is used to prepare the input for processing. A strict resource limiting program called TreeLimitedRun is used to limit the CPU time and memory used by an ATP system or tool. TreeLimitedRun monitors processes' resource usage more tightly than is possible with standard operating system calls. Finally a program called X2tptp converts an ATP system's output to TPTP format, if requested by the user. 
The web interfaces SystemB4TPTP, SystemOnTPTP, and SystemOnTSTP provide interactive online access to the SystemOnTPTP utility. ${ }^{3}$ The online service can also be accessed directly with http POST requests. The SystemB4TPTP interface provides access to tools for preparing problems for submission to an ATP system, including conversion from other (non-TPTP) formats to TPTP format, parsing and syntax checking, type checking, and pretty printing. In addition to providing access to ATP systems, the SystemOnTPTP interface additionally provides system reports, recommendations for systems to use on a given problem, and direct access to the SSCPA system [26] that runs multiple systems in competition parallel. The SystemOnTSTP interface provides access to solution processing tools, including parsing and syntax checking, pretty printing, derivation verification using GDV [23], interactive graphical proof presentation using IDV [28], answer extraction [27], and proof conversion and summarization tools. The three interfaces have options to pass the output from a system/tool on to the next interface - from problem preparation, to problem solving, to solution processing. The output is returned to browsers in appropriate HTML wrapping, and can also be obtained in its raw form for processing on the client side (typically when the interfaces are called programmatically using http POST requests). The online service is hosted at the University of Miami on a server with four $2.33 \mathrm{GHz}$ CPUs, 4GB RAM, and running the Linux 2.6 operating system.

\section{MizAR}

MizAR is running experimentally on our server ${ }^{4}$, where it can be best learned by exploration. A good way to explore is to start with an existing simple Mizar article, e.g., the card_1 article $^{5}$ about cardinal numbers $[1]^{6}$, from the MML. ${ }^{7}$ Within MizAR, select the "URL to fetch article from" field, insert the article's URL into the text box, and press the "Send" button. For experienced Mizar users, there is also a simple way to send the current Mizar buffer to the remote service, by running the mizar-post-to-ar4mizar interactive function in the Mizar mode for Emacs [30]. Both actions call the main MizAR cgi-bin script with appropriate arguments, which launches the functions described below.

MizAR links together a number of Mizar and ATP-related components, which are useful for general work with formal mathematics in Mizar. The main components are as follows (details are provided in the rest of Section 3):

- Web access to the whole cross-linked HTMLized MML.

- Fast server-based verification of a Mizar article.

- Disambiguation of the article by HTMLization, producing an HTML presentation of the verified article with links to an HTMLized version of the

\footnotetext{
${ }^{3}$ Available starting at http://www.tptp.org/cgi-bin/SystemOnTPTP

${ }^{4}$ http://mws.cs.ru.nl/ mptp/MizAR.html

${ }^{5}$ http://mws.cs.ru.nl/ mptp/mml/mml/card_1.miz

${ }^{6}$ Available at http://mizar.uwb.edu.pl/JFM/Vol1/ordinal1.html

7 All articles are available from http://mws.cs.ru.nl/ mptp/mml/mml
} 
MML. Additional useful information is also extracted during HTMLization, and included in the HTML presentation of the article.

- Fast translation of the article to MPTP (Mizar Problems for Theorem Provers) format.

- Fast generation of ATP problems in TPTP format, for all the theorems in the article, and for all the atomic inferences done by Mizar.

- Easy access to default ATP systems for solving the ATP problems, and access to SystemOnTPTP for solving more difficult problems.

- Easy access to IDV for visualization and postprocessing of proofs found by the ATP systems.

- Suggesting useful hints for proving (either by ATP or interactively in Mizar) particular Mizar lemmas and theorems.

Figure 1 shows the overall structure of the MizAR system. The leftmost column shows the various forms of the article that are produced, and the two bold boxes in the next column are the HTML presentations for user interaction. The third column shows the software tools that generate the various dataforms, using the article and the background information shown in the rightmost column. A Mizar article is submitted through the web interface or from Emacs. The article is then verified and converted to XML format, which is subsequently rendered in HTML format with links to the MML. The HTML presentation includes links that allow the user to proceed with further processing, and is the main interface for user interaction with MizAR. While the HTML is presented to the user, the article is asynchronously converted to the MPTP format, which is used for generating TPTP format ATP problems. The ATP problems can then be submitted to ATP systems, either locally via SystemOnTPTP. The ATP systems' solutions are used to enrich the HTML presentation, and can be passed on to various post-processing tools. The subcomponents that perform these tasks are described in more detail below.

\subsection{Server-based Verification of a Mizar Article}

Unlike many other (especially LCF-inspired) proof assistants, Mizar is a compilerlike batch processor, verifying a whole article in one pass. While a lot of work on Mizar goes into balancing the strength, speed, and obviousness of the proof checking, the process of checking a whole article can get quite time-consuming for longer and more complex articles, especially on older hardware.

There are several advantages to remote server-based verification of Mizar articles. The first obvious advantage is that having everything web-based removes the need for a local installation of Mizar. The second advantage is that even if Mizar is installed locally, it is often more convenient to quickly (try to) verify an article in a browser, instead of launching the verification environment on one's local computer. In cases when the article is available online, it is possible to provide that URL as an argument to the MizAR URL, to directly launch MizAR on the article. ${ }^{8}$ This makes such verification snippets available in all kinds of

\footnotetext{
${ }^{8}$ For example, http://mws.cs.ru.nl/ mptp/cgi-bin/MizAR.cgi?ProblemSource= URL\&FormulaURL=http://mws.cs.ru.nl/ mptp/mml/mml/card_1. miz\&Name=Test1
} 


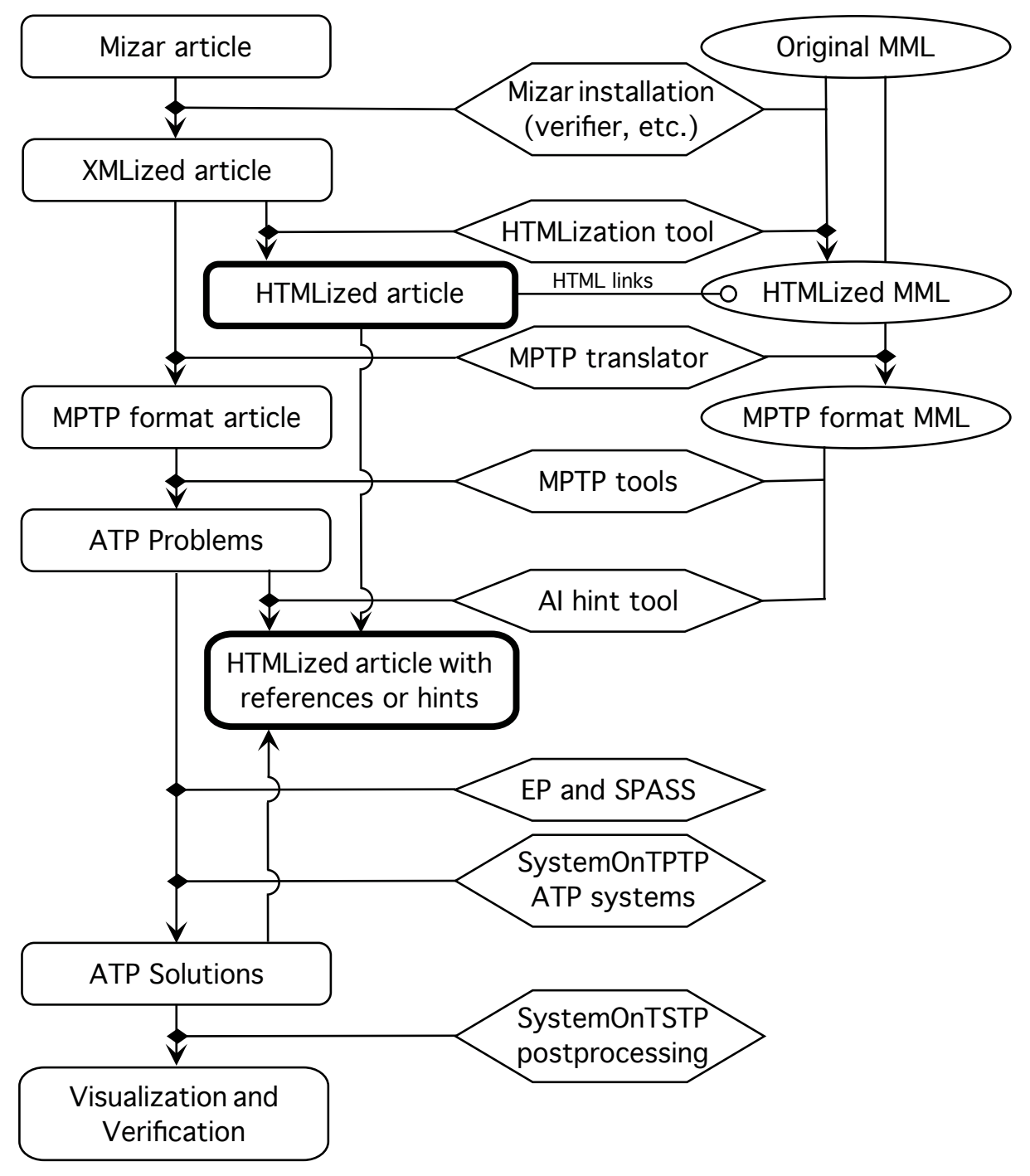

Fig. 1. Structure of the MizAR system

online fora (e-mail discussions, blog and twitter posts, wikis, etc.) with direct rendering of the results. In short, the third advantage is that a web service provides mechanism for online communication of verification results.

The fourth (and probably greatest) advantage of server-based verification is the raw verification speed. A dedicated server usually runs on reasonably new hardware with enough memory, etc. For example, even for the relatively short card_1 Mizar article mentioned above, full verification on a recent notebook 
(1.66 GHz Intel Atom) takes $2 \mathrm{~s}$, while on a recent lower-range server (2 quadcore hyperthreading $2.27 \mathrm{GHz}$ Intel Xeons) the same task takes $0.5 \mathrm{~s}$. For a more involved article this difference becomes more visible, and can be the deciding factor for the usability and real-time experience with the system. For example, for the more involved Mizar article fdiff_ $1^{9}$ about real function differentiability [19], the difference is $23 \mathrm{~s}$ vs. $6 \mathrm{~s}$.

The latest advances in CPU power have been achieved mainly by packing multiple CPUs together, instead of raising the speed of individual CPUs. To take advantage of this, Mizar processing has recently been parallelized ${ }^{10}$, and the parallel version of the Mizar verifier is running on our server. This (depending on the success of the parallelization) can further significantly improve the real-time verification experience. For example, on the even longer Mizar article $f$ diff $\_2^{11}$ about real function differentiability [13], the difference between running the parallel version (using eight cores) and the non-parallel version is a factor of four (31s vs. 7.8s). Verification of this article using the above mentioned notebook takes $125 \mathrm{~s}$, resulting in a speed-up factor of sixteen (and substantially improving the real-time interaction with the system).

The last important advantage is that a server-based installation supports use of modified, enhanced, and experimental versions of the verifier. This can provide useful additional functionalities. For instance, the Mizar parallelizer requires additional software to run, and a recently modified version of the Mizar verifier that has not yet been distributed to Mizar users. Translation of Mizar articles to ATP formats also requires a version of the verifier that has been compiled with a special flag, again not included in the standard Mizar distribution. An online service can also easily include multiple versions of the Mizar library and binaries, as is done for the MML Query service [2].

\subsection{HTMLization of Mizar Articles}

There has been quite a lot of recent work on XMLization and HTMLization of Mizar articles $[29,35]$, including the addition of useful additional information into the XML form of the Mizar article and its HTML presentation. There are two major reasons for having a static HTMLized MML available: ${ }^{12}$ (i) to provide fast browsing of the theorems and definitions used in a particular formalization, with a number of user-friendly features (like (sub)proof hiding/showing, etc.), and (ii) providing explanations for a number of phenomena in the formalization that are made explicit and clear only during verification, and are hard to decipher from the formalization text alone. The latter includes, for example:

- Explicit HTML presentation of the current goal (thesis), computed by the verifier at each point of the formalization.

\footnotetext{
${ }^{9}$ http://mws.cs.ru.nl/ mptp/mml/mml/fdiff_1.miz

10 The description of the Mizar parallelization and related experiments is unpublished as of January 2010. The parallelizer is available at http://github.com/JUrban/ MPTP2/raw/master/MizAR/cgi-bin/bin/mizp.pl.

${ }^{11}$ http://mws.cs.ru.nl/ mptp/mml/mml/fdiff_2.miz

${ }^{12}$ It is available at http://mws.cs.ru.nl/ mptp/mml/html/
} 
- Proper disambiguation of overloaded mathematical symbols. Overloading is necessary in a large body of mathematics including all kinds of subfields, but at the same time makes it difficult for readers of the textual versions of the articles to understand the precise meaning of the overloaded symbols.

- Explicit access to formulae for definition correctness, and formulae expressing properties (projectivity, antisymmetry, etc.) that are computed by the verifier. Making these explicit in the HTML presentation can help users.

- Explicit representation of other features that are implicit in Mizar verification, e.g., definitional expansions, original versions of constructors that have been redefined, etc. Making these explicit in the HTML presentation can also help users.

The static HTMLized MML is an important resource used by MizAR. The articles submitted to MizAR are dynamically linked to the static HTMLized MML. This is a notable difference to SystemOnTPTP, which treats each problem as an independent entity. The first implementation of MizAR has focused on developing the services for a fixed version of the MML. However, management of library versions is a nontrivial and interesting problem. Allowing users to verify new articles and also refactor existing ones will ultimately lead into the area of formal mathematical wikis $[6,5]$.

The main functions of the HTMLization service are to (i) provide quick linking to the static HTMLized MML (thus providing the disambiguation and explanation functions described above), and (ii) allow a number of additional (mainly automated reasoning and AI) services to be launched by suitable CGI and AJAX calls from links in the HTML. These additional services are described in the following subsections. The main features of server-side HTMLization are increased speed, and the availability of additional programs and features. While the Mizar HTML processing was designed to be locally available, using just a browser-based XSL processor (i.e., loading the XML produced by Mizar directly into a browser, which applies the appropriate style sheet), even the basic XSL processing in the browser can take a long time (minutes). Again, having a specialized fast XSL processor installed on the server helps quite a lot, and the HTMLization can be parallelized using techniques similar to the parallelization of the basic verification process. This provides a much better HTMLization response, and also makes additional XSL-based preprocessing possible. This is needed for better HTML quality, and for the translation to ATP formats.

\subsection{Generation of ATP Problems in TPTP Format}

One of the main objectives of MizAR (as suggested by its name) is to allow easy experimentation with ATP systems over the large body of formal mathematics available in the MML, and to apply ATP functionalities on Mizar articles. The MPTP system [32,31] for translating Mizar articles to the TPTP format has been modified to work in a fast real-time mode, generating ATP problems corresponding to Mizar proof obligations. The MPTP system translates Mizar articles to the MPTP format, which is an extension of the TPTP format with 
information needed for further processing into ATP problems. Like the static HTMLized MML, a static copy of the MML in MPTP format is available to MizAR. It is used for building translated MML items (theorems, definitions, formulae encoding Mizar type automations, etc.) that are necessary for creating complete ATP problems.

Using MPTP and generating ATP problems requires a quite complex installation and setup (SWI Prolog, Unix, special XSL style sheets, the translated MML in the MPTP format, etc.), so this is a good example of an additional functionality that would be quite hard to provide locally. The MPTP was initially designed for offline production of interesting ATP problems and data, and was not optimized for speed. Several techniques have been used to provide a reasonable real-time experience:

- More advanced (graph-like) data structures have been used to speed up the selection of parts of the MML necessary for generating the ATP problems.

- Larger use has been made of Prolog indexing and the asserted database, for various critical parts of the code.

- The MPTP version of the MML has been factored so that it is possible to work with only the parts of the MML needed for a given article.

These techniques have led to reasonable real-time performance of the MPTP problem generation, comparable to the performance of Mizar verification and HTMLization. For example, the MPTP processing followed by the generation of all 586 ATP problems for the card_1 article takes $7 \mathrm{~s}$ on the server.

After the conversion to MPTP format, the ATP problems for an article are generated asynchronously while the user is presented with the HTMLized article. There is a small danger of the user wanting to solve an ATP problem that has not yet been generated. However, it is easy to check if the translation process is finished, and which ATP problems are already available, by examining the system log.

The MPTP processing has not been parallelized (like the Mizar verification and HTMLization). However, there are no serious obstacles to that. Another speed-up option would be to ask MPTP to generate only a subset of the ATP problems (this is actually how the parallelization is going to work), selected in some reasonable way by the formalizer in the user interface. It is also possible to keep the MPTP system loaded and listening once it has generated a subset of problems, and to have it generate new ATP problems from the current article on demand.

The HTMLization of an article and the generation of ATP problems are independent processes that could be separated into two separate services. Users might, for instance, be interested only in HTML-like disambiguation of their articles, or only in getting explanations and advice from ATP systems, without looking at the HTML form of the article. With sufficient CPU-cores in the server, none of these two possible use-cases suffers in terms of the response time. 


\subsection{Calling ATP Systems}

The calling of ATP systems to solve the ATP problems is built into the HTML presentation of the user's article, by linking the available ATP services to keywords in the HTML presentation. This follows the general idea that the HTML serves as the main interface for calling other services. The links to the ATP services in the HTML are the Mizar keywords by and from, indicating semantic justification in Mizar. For example, the Mizar justification

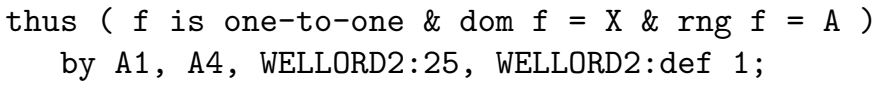

in the last line of the proof of theorem Th4 in the card_1 article says that the Mizar checker should be able to verify that the formula on the left hand side of the by keyword follows from the previously stated local propositions, theorems and definitions A1, A4, WELLORD2:25, WELLORD2: def 1, and some knowledge that the Mizar verifier uses implicitly. There are now the following use-cases calling ATP systems:

1. Mizar has verified the inference, possibly using some implicit information. The user is interested in knowing exactly what implicit information was used by Mizar, and exactly how the proof was conducted.

2. Mizar has not verified the inference. The user is interested in knowing if the inference is logically valid, if it can be proved by a (stronger) ATP system, and what such an ATP proof looks like.

The first use-case typically happens for one of two reasons. The first reason is that the theory in which the author is working has become very rich, and involves many implicit (typically typing) Mizar mechanisms that make the formal text hard to understand. The TPTP translation has to make all this implicit information explicit in the TPTP problems, and the resulting corresponding ATP proofs show explicitly how this information is used. For the Mizar justification above, clicking on the by keyword calls the EP system [20] on the ATP problem, with a several second time limit. If a proof is found, the interface is refreshed with an explanation box that includes (among other things described below) a list of the references used in the proof, as shown in Figure 2. In this case the exact references shown to the user are following:

e8_9_-mtest1, dt_k1_wellord2, dt_c2_9__mtest1, e2_9__mtest1, e7_9__mtest1, t25_wellord2, d1_wellord2

These references use the MPTP syntax, but are linked (dynamically using AJAX calls) to the corresponding places in the theorem's HTML or the static HTMLized MML), and are given appropriate explanation titles. Note that the EP proof uses seven more references than the four that are in the original Mizar by inference. One reference is added because it explicitly denotes the formula being proved (the left-hand side of by), and the two remaining references encode implicit type declarations that are used by Mizar (the type of the local constant R, 


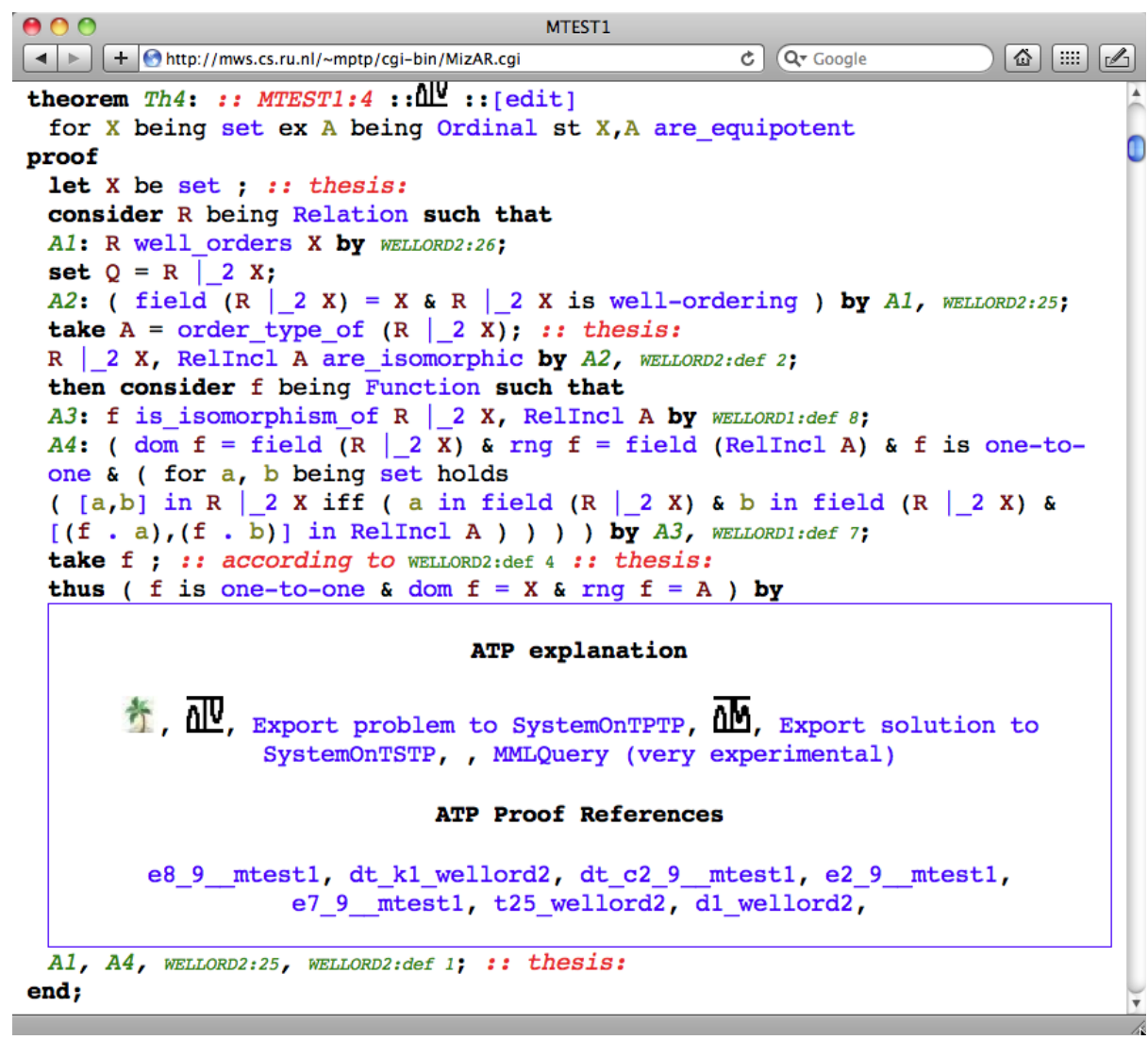

Fig. 2. ATP explanation box

and the type of the functor Relincl that is used in proposition A4 (renamed to e7_9_mtest1 by MPTP)). The ATP proof can be visualized in the IDV system by clicking on the palm tree icon in the explanation box.

The second reason for the first use-case is cross-verification. In cases when a bug in the Mizar implementation is suspected, or incompleteness in the ATP translation is suspected, the user may be interested in knowing if the Mizar proof can be done by another system (and how). In this sense the environment is used for gathering additional information and debugging. The cross-verification rates for Mizar justifications are reasonably high [33], which makes this usage realistic.

The second use-case (finding proofs that are too hard for Mizar) is the real "ATP proof assistance" dream, i.e., using ATP systems to automatically find proofs for ITPs. Users can do this within MizAR by providing a large set of "potentially relevant" Mizar propositions on the right-hand side of the by keyword, and letting the EP system try to find a proof. Note that if EP does not find the problem to be countersatisfiable, the user also has the option to try the SPASS 
ATP system [36] directly from the interface, as shown in Figure 3. This is justified by the general experience that SPASS is reasonably complementary to EP when solving MPTP problems. If SPASS is not successful the user can use the links and icons in the explanation box to inspect the ATP problem, and launch the SystemOnTPTP interface to try the ATP systems available there. The proofs found by the ATP systems can be processed in the SystemOnTSTP interface, including visualization using the IDV system, analysis using the AGInT system for finding the interesting steps in proofs, and ATP-based cross-verification using the GDV verifier.

\subsection{Getting Hints for Necessary Mizar References}

If none of the ATP systems can find a proof for an ATP problem (corresponding to a Mizar inference), either because the ATP system timed out or found that the ATP problem is countersatisfiable (as in Figure 3, then typically some more assumptions (Mizar references) have to be added to the TPTP problem. The explanation box can provide hints for proving the Mizar proposition. This is done using the "Suggest hints" link that is put into the box when EP fails to find a proof (see Figure 3). The "Suggest hints" button is linked to a Bayesian advisor that has been trained on the whole MML (i.e., on all of the proofs in it). (See [34] for the details of how the machine learning is organized in the context of a large deductive repository like MML. Other axiom selection systems could be used in a similar way.) The trained advisor runs as a daemon on the web server, and receives queries initiated by clicking on the "Suggest hints" button. This service is very fast, and the hints are usually provided in milliseconds. They

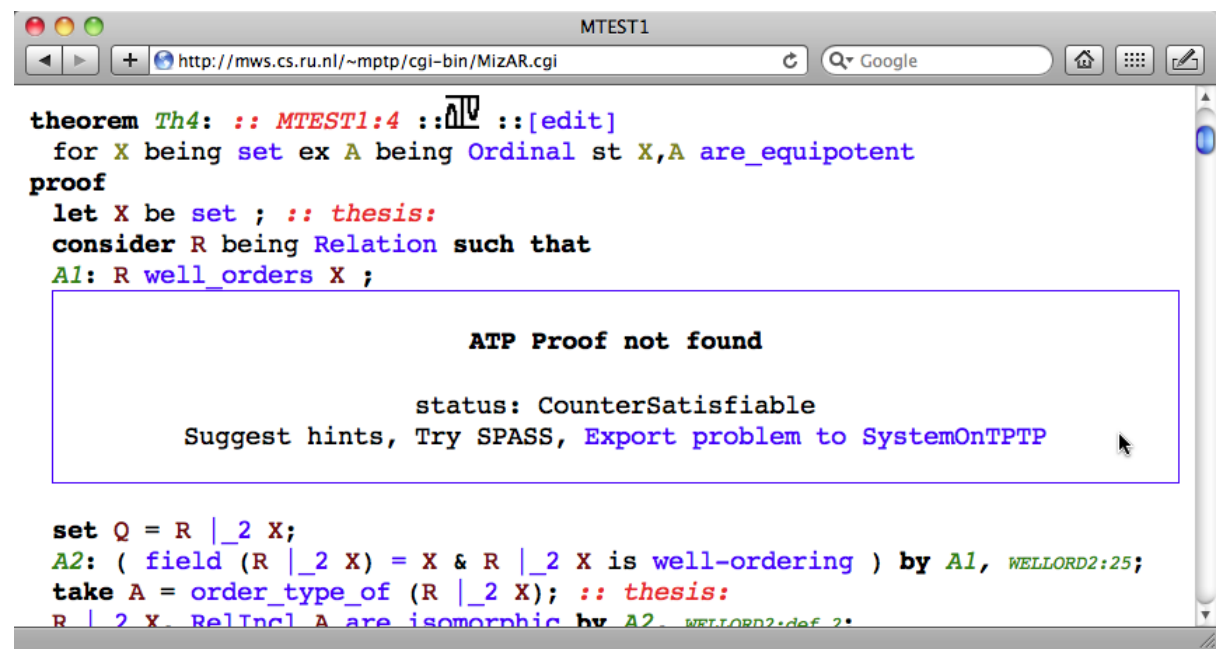

Fig. 3. ATP explanation box for "Proof not found" 
are HTMLized and inserted (by AJAX calls) into the explanation box, as shown in Figure 4.

\section{Future Work and Conclusions}

This paper has introduced the MizAR web service that allows Mizar users to use automated reasoning tools on their Mizar articles. MizAR is to some degree based on and similar to the SystemOnTPTP service for solving first-order ATP problems. The main differences to SystemOnTPTP are the use of the Mizar language that is oriented towards human mathematicians (rather than the pure first-order logic used in SystemOnTPTP), and setting the service in the context of the large Mizar Mathematical Library of previous theorems, definitions, and proofs.

There are obvious differences to those systems, given by the large-theory setting in which Mizar formalization is typically done. There are several use-cases described above, ranging from using HTMLization to disambiguate complicated Mizar syntax, usage of ATP systems to explain Mizar inferences, provide new proofs, and find counterexamples, to using additional AI-based services for proof advice, like the proof advisor trained on the whole MML.

There are many directions for future work in this setting, some of them mentioned above. The service already is available by an interactive call from the

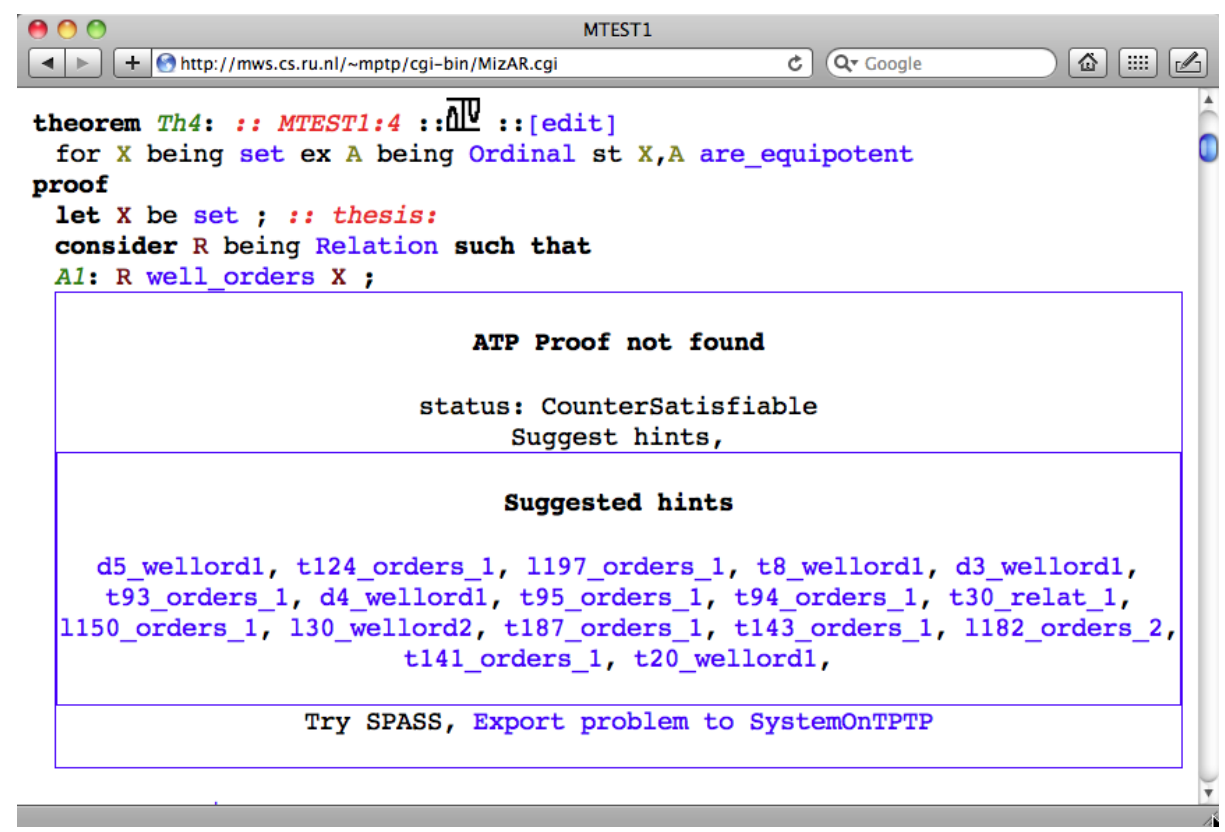

Fig. 4. ATP explanation box offering hints 
Emacs interface. However, Emacs simply generates the request from the current Mizar buffer, and lets the user see the response (and all the associated functionalities) in a browser. Obviously, the ATP and proof advising functionalities could be made completely separate from the HTML presentation, and sent directly to the Emacs session.

As mentioned above, the static MML is now present on the server in both HTML and MPTP format (and obviously in raw text and in the Mizar internal format), but not directly editable by the users. Giving the user the ability to edit the supporting MML forms leads in the direction of formal mathematical wikis, with all the interesting persistence, versioning, linking, user-authentication, and dependency problems to solve. There is an experimental ikiwiki-based prototype available for Mizar and the MML, which solves some of the persistence and user-authentication problems, and that is likely to be merged with the services presented here. Hopefully this will form a rich wiki for formal mathematics, with a large number of services providing interesting additional functionalities to people interested in formal mathematics.

There is also a large amount of work that can be done on making the system nicer and more responsive, e.g., the parallelization of the MPTP processing is

yet to be done, better machine learning and hint suggestion methods can be used and linked to the ATP services, and presentations in formats other than HTML (e.g., TeX and PDF are also used for Mizar) would be nice to include.

\section{References}

1. G. Bancerek. The Ordinal Numbers. Journal of Formalized Mathematics, 1(1):9196, 1990.

2. G. Bancerek and P. Rudnicki. Information Retrieval in MML. In A. Asperti, B. Buchberger, and J.H. Davenport, editors, Proceedings of the 2nd International Conference on Mathematical Knowledge Management, number 2594 in Lecture Notes in Computer Science, pages 119-132. Springer-Verlag, 2003.

3. C. Benzmüller and L. Paulson. Multimodal and Intuitionistic Logics in Simple Type Theory. Logic Journal of the IGPL, 2010.

4. S. Conchon, E. Contejean, J. Kanig, and S. Lescuyer. Lightweight Integration of the Ergo Theorem Prover Inside a Proof Assistant. In J. Rushby and N. Shankar, editors, Proceedings of the 2nd Workshop on Automated Formal Methods, pages 55-59. ACM Press, 2007.

5. P. Corbineau, H. Geuvers, C. Kaliszyk, J. McKinna, and F. Wiedijk. A Real Semantic Web for Mathematics Deserves a Real Semantics. In C. Lange, S. Schaffert, H. Skaf-Molli, and M. Völkel, editors, Proceedings of the 3rd Semantic Wiki Workshop, number 360 in CEUR Workshop Proceedings, pages 62-66, 2008.

6. P. Corbineau and C. Kaliszyk. Cooperative Repositories for Formal Proofs. In M. Kauers, M. Kerber, R. Miner, and W. Windsteiger, editors, Proceedings of the 6th International Conference on Mathematical Knowledge Management, volume 4573 of Lecture Notes in Computer Science, pages 221-234, 2007.

7. V. D'Silva, D. Kroening, and G. Weissenbacher. A Survey of Automated Techniques for Formal Software Verification. IEEE Transactions on Computer-aided Design of Integrated Circuits and Systems, 27(7):1165-1178, 2008. 
8. G. Gonthier. Formal Proof - The Four-Color Theorem. Notices of the American Mathematical Society, 55(11):1382-1393, 2008.

9. T. Hales. A Proof of the Kepler Conjecture. Annals of Mathematics, 162(3):10651185, 2005.

10. T. Hales, editor. A Special Issue on Formal Proof, volume 55, 2008.

11. J. Hurd. First-Order Proof Tactics in Higher-Order Logic Theorem Provers. In M. Archer, B. Di Vito, and C. Munoz, editors, Proceedings of the 1st International Workshop on Design and Application of Strategies/Tactics in Higher Order Logics, number NASA/CP-2003-212448 in NASA Technical Reports, pages 56-68, 2003.

12. G. Klein, K. Elphinstone, G. Heiser, J. Andronick, D. Cock, P. Derrin, D. Elkaduwe, K. Engelhardt, R. Kolanski, M. Norrish, T. Sewell, H. Tuch, and S. Winwood. seL4: Formal Verification of an OS Kernel. In T. Anderson, editor, Proceedings of the 22nd ACM Symposium on Operating Systems Principles, pages 207-220. ACM Press, 2009.

13. J. Kotowicz and K. Raczkowski. Real Function Differentiability - Part II. Formalized Mathematics, 2(3):407-411, 1991.

14. W.W. McCune. Solution of the Robbins Problem. Journal of Automated Reasoning, 19(3):263-276, 1997.

15. L. Paulson. A Generic Tableau Prover and its Integration with Isabelle. Artificial Intelligence, 5(3):73-87, 1999.

16. A. Pease and G. Sutcliffe. First Order Reasoning on a Large Ontology. In J. Urban, G. Sutcliffe, and S. Schulz, editors, Proceedings of the CADE-21 Workshop on Empirically Successful Automated Reasoning in Large Theories, number 257 in CEUR Workshop Proceedings, pages 59-69, 2007.

17. J.D. Phillips and D. Stanovsky. Automated Theorem Proving in Loop Theory. In G. Sutcliffe, S. Colton, and S. Schulz, editors, Proceedings of the CICM Workshop on Empirically Successful Automated Reasoning in Mathematics, number 378 in CEUR Workshop Proceedings, pages 42-53, 2008.

18. Y. Puzis, Y. Gao, and G. Sutcliffe. Automated Generation of Interesting Theorems. In G. Sutcliffe and R. Goebel, editors, Proceedings of the 19th International FLAIRS Conference, pages 49-54. AAAI Press, 2006.

19. K. Raczkowski and P. Sadowski. Real Function Differentiability. Formalized Mathematics, 1(4):797-801, 1990.

20. S. Schulz. E: A Brainiac Theorem Prover. AI Communications, 15(2-3):111-126, 2002.

21. M. Suda, G. Sutcliffe, P. Wischnewski, M. Lamotte-Schubert, and G. de Melo. External Sources of Axioms in Automated Theorem Proving. In B. Mertsching, editor, Proceedings of the 32nd Annual Conference on Artificial Intelligence, number 5803 in Lecture Notes in Artificial Intelligence, pages 281-288, 2009.

22. G. Sutcliffe. SystemOnTPTP. In D. McAllester, editor, Proceedings of the 17th International Conference on Automated Deduction, number 1831 in Lecture Notes in Artificial Intelligence, pages 406-410. Springer-Verlag, 2000.

23. G. Sutcliffe. Semantic Derivation Verification. International Journal on Artificial Intelligence Tools, 15(6):1053-1070, 2006.

24. G. Sutcliffe. The TPTP Problem Library and Associated Infrastructure. The FOF and CNF Parts, v3.5.0. Journal of Automated Reasoning, 43(4):337-362, 2009.

25. G. Sutcliffe, S. Schulz, K. Claessen, and A. Van Gelder. Using the TPTP Language for Writing Derivations and Finite Interpretations. In U. Furbach and N. Shankar, editors, Proceedings of the 3rd International Joint Conference on Automated Reasoning, number 4130 in Lecture Notes in Artificial Intelligence, pages 67-81, 2006. 
26. G. Sutcliffe and D. Seyfang. Smart Selective Competition Parallelism ATP. In A. Kumar and I. Russell, editors, Proceedings of the 12th International FLAIRS Conference, pages 341-345. AAAI Press, 1999.

27. G. Sutcliffe, A. Yerikalapudi, and S. Trac. Multiple Answer Extraction for Question Answering with Automated Theorem Proving Systems. In H. Guesgen and C. Lane, editors, Proceedings of the 22nd International FLAIRS Conference, pages 105-110. AAAI Press, 2009.

28. S. Trac, Y. Puzis, and G. Sutcliffe. An Interactive Derivation Viewer. In S. Autexier and C. Benzmüller, editors, Proceedings of the 7th Workshop on User Interfaces for Theorem Provers, 3rd International Joint Conference on Automated Reasoning, volume 174 of Electronic Notes in Theoretical Computer Science, pages 109-123, 2006.

29. J. Urban. XML-izing Mizar: Making Semantic Processing and Presentaion of MML Easy. In M. Kohlhase, editor, Proceedings of the 4th International Conference on Mathematical Knowledge Management, number 3863 in Lecture Notes in Computer Science, pages 346-360, 2005.

30. J. Urban. MizarMode - An Integrated Proof Assistance Tool for the Mizar Way of Formalizing Mathematics. Journal of Applied Logic, 4(4):414-427, 2006.

31. J. Urban. MPTP 0.2: Design, Implementation, and Initial Experiments. Journal of Automated Reasoning, 37(1-2):21-43, 2006.

32. J. Urban. Automated Reasoning for Mizar: Artificial Intelligence through Knowledge Exchange. In G. Sutcliffe, P. Rudnicki, R. Schmidt, B. Konev, and S. Schulz, editors, Proceedings of the LPAR Workshops: Knowledge Exchange: Automated Provers and Proof Assistants, and The 7th International Workshop on the Implementation of Logics, number 418 in CEUR Workshop Proceedings, pages 1-16, 2008.

33. J. Urban and G. Sutcliffe. ATP-based Cross Verification of Mizar Proofs: Method, Systems, and First Experiments. Journal of Mathematics in Computer Science, 2(2):231-251, 2009.

34. J. Urban, G. Sutcliffe, P. Pudlak, and J. Vyskocil. MaLARea SG1: Machine Learner for Automated Reasoning with Semantic Guidance. In P. Baumgartner, A. Armando, and D. Gilles, editors, Proceedings of the 4 th International Joint Conference on Automated Reasoning, number 5195 in Lecture Notes in Artificial Intelligence, pages 441-456. Springer-Verlag, 2008.

35. J. Urban, G. Sutcliffe, S. Trac, and Y. Puzis. Combining Mizar and TPTP Semantic Presentation and Verification Tools. Studies in Logic, Grammar and Rhetoric, 18(31):121-136, 2009.

36. C. Weidenbach, R. Schmidt, T. Hillenbrand, R. Rusev, and D. Topic. SPASS Version 3.0. In F. Pfenning, editor, Proceedings of the 21st International Conference on Automated Deduction, number 4603 in Lecture Notes in Artificial Intelligence, pages 514-520. Springer-Verlag, 2007. 